\title{
The role of auxin transporters and receptors in adventitious rooting of Arabidopsis thaliana pre-etiolated flooded seedlings
}

\author{
Cibele Tesser da Costa ${ }^{\mathrm{a}}$, Remko Offringa ${ }^{\mathrm{b}, *}$, Arthur Germano Fett-Neto ${ }^{\mathrm{a} \text {,* }}$ \\ ${ }^{a}$ Plant Physiology Laboratory, Center for Biotechnology and Department of Botany, Federal University of Rio Grande do Sul (UFRGS), CP 15005, Porto Alegre, RS, 91501- \\ 970, Brazil \\ ${ }^{\mathrm{b}}$ Department of Plant Developmental Genetics, Institute of Biology Leiden, Sylvius Laboratory, Sylviusweg 72, 2333 CB, Leiden, the Netherlands
}

\section{A R T I C L E I N F O}

\section{Keywords:}

Adventitious roots

Auxin transport

Arabidopsis

Mutant

Reporter gene

PIN phosphorylation

\begin{abstract}
A B S T R A C T
Adventitious roots (ARs) form from above-ground organs, and auxins are major regulators of AR development. TIR1/AFB F-box proteins act as well-established auxin receptors. Auxin transport involves the PINFORMED (PIN) auxin efflux carriers and AUXIN RESISTANT 1/LIKE AUX1 (AUX1/LAX1) influx carriers. To further elucidate the basis of AR development, we investigated the participation of these proteins and phosphorylation of PINs during adventitious rooting in hypocotyls of pre-etiolated flooded Arabidopsis thaliana seedlings. Mutant and GUS localization studies indicated that AFB2 is important in AR development. AUX1 loss-of-function reduced AR numbers, which could not be reversed by exogenous auxin. Single mutations in $L A X 1, L A X 2$ and $L A X 3$ had no negative impact on AR development and the first and last mutations even promoted it. Double and triple mutants of AUX1, LAX1, LAX2 and LAX3 significantly reduced rooting, which was reversed by exogenous auxin. AUX1 was essential in AR establishment, with LAX3 apparently acting in conjunction. Proper phosphorylation of PINs by PID, WAG1 and WAG2 and auxin transport direction were equally essential for AR establishment. PIN1, $A U X 1$ and AFB2 (overexpression) and LAX1, LAX3, PIN4 and PIN7 (downregulation) emerged as potential targets for genetic manipulation aiming at improving AR development.
\end{abstract}

\section{Introduction}

The root system of a plant has several essential roles, such as uptake of water and nutrients and anchorage in the soil [1]. Root system architecture can be modulated in response to environmental cues as a plant strategy to adapt and survive. This modulation can include root growth and development of lateral roots (LRs) and adventitious roots (ARs) (reviewed by [2]). The main difference between LRs and ARs is that the former develop from the pericycle of existing roots, whereas ARs are established from organs other than roots [3]. ARs may naturally form from stem cells or their development can be stimulated by wounding or hormonal application. Their shallow growth angle can increase phosphorus uptake in upper soil strata, for example [1,4]. ARs are also important for vegetative propagation of plants, being essential to produce clones of economically important plant species. However, for several of these plant species vegetative propagation is limited by the low efficiency of AR formation on stem cuttings or regenerated shoots. ARs and LRs share some common mechanisms and steps in their development, but differences between them are expected and not fully understood to date $[4,5]$.

Several plant hormones are known to participate in the different steps of AR formation, and auxins, such as indole-3-acetic acid (IAA), are recognized as the main hormones regulating this process [6,7]. Auxin perception by the cell leads to changes in gene expression through the Auxin Response Factors (ARFs). At low auxin concentrations these ARFs are bound by Aux/IAA transcriptional repressors, and this inhibits transcription of auxin responsive genes. In the presence of auxin, the TIR1/AFB F-box proteins, which are part of the $S \mathrm{CF}^{\mathrm{TIR} 1 / \mathrm{AFB}}$ (Skp1/Cullin/F-box) E3 ubiquitin ligase complex, recruit the Aux/IAA transcriptional repressors to promote their ubiquitination and subsequent degradation [8-10]. This subsequently frees the ARFs and allows them to activate transcription [11-13].

The auxin level in the cell is determined by its biosynthesis, turnover and transport. Auxin biosynthesis occurs in the young developing

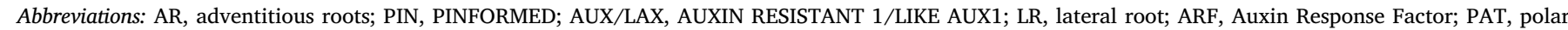

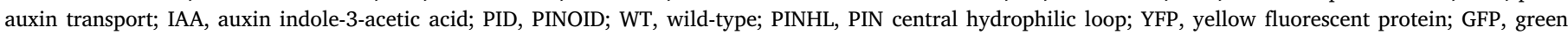
fluorescent protein; GUS, $\beta$-glucuronidase

* Corresponding authors.

E-mail addresses: r.offringa@biology.leidenuniv.nl (R. Offringa), fettneto@cbiot.ufrgs.br (A.G. Fett-Neto).
} 
organs in the shoot, and from there the hormone is polarly transported from cell to cell, first to the shoot apex where auxin maxima are initiation points of new organs, and subsequently down the stem to the root system. This polar auxin transport (PAT) is orchestrated through the action of three protein families, the AUXIN RESISTANT 1/LIKE AUX1 (AUX/LAX) influx carriers, the PIN-FORMED (PIN) efflux carriers and the P-GLYCOPROTEIN (PGP/ABCB) transporters [14,15].

The PIN protein family in Arabidopsis comprises eight members that can be divided in two groups: (1) the PIN1-type proteins (PIN1, 2, 3, 4, and 7) that are the main drivers of PAT as they determine the direction of transport through their asymmetric distribution at the plasma membrane and, (2) the PIN5-type proteins (PIN5, 6 and 8) that are localized in the endoplasmic reticulum and are involved in controlling the nuclear auxin homeostasis [16]. The polar distribution of the PIN1type carriers was shown to be determined by reversible phosphorylation of central serines in three conserved TPRXS(N/S) motifs in the PIN central hydrophilic loop (PINHL) through the antagonistic action of the protein Ser/Thr kinases PINOID (PID), WAG1 and WAG2 and protein phosphatase 2A (PP2A) [17-19]. PIN proteins phosphorylated by these kinases accumulate at the shootward (apical) side of cells in embryos or the root and shoot meristem, whereas de-phosphorylation by PP2A leads to their rootward (basal) localization.

Auxin transporters regulate several aspects of root growth and morphology. PIN3 and PIN7 redirect auxin flux in the root in response to gravity stimulation and establish the asymmetric growth of plants [20,21]. PIN3 and PIN7 have their expression increased by ethylene application on Arabidopsis seedlings and LR formation is blocked [22], possibly by preventing the required auxin maxima establishment needed for LR development. PIN1 acts in the establishment of a root tip auxin maxima together with LAX3, positioning the quiescent center in ARs [23]. The efflux protein PIN4 is important for auxin homeostasis and patterning of the root tip [24], whereas its participation in AR development is still unknown.

The aux 1 mutant has a reduced number of lateral roots and it was shown that AUX1 plays important roles in LR development through auxin transport between source and sink tissues [25]. The influx carrier LAX3 is induced by auxins and promotes LR emergence [26]. The spatial pattern of expression of LAX3 is affected by the efflux auxin transporter PIN3 in LRs, contributing to an auxin flux to cells overlying LR primordia [27]. Sequential induction of PIN3 and LAX3 causes softening of some cells and contributes to LR emergence [27]. In ARs, PIN1 and LAX3 establish an auxin maximum in the root tip, thereby restricting the expression of WOX5 and delimiting the quiescent center [23].

To further understand the role of auxin signaling and transport in AR development in Arabidopsis, we tested different mutants in genes encoding auxin receptors, or influx and efflux transporters for their efficiency in AR development, in the pre-etiolated flooded seedling model. This identified key players in ARs, among which auxin receptors and transporters, providing clues as to which target genes to silence or overexpress in order to stimulate AR development.

\section{Material and methods}

\subsection{Plant material, growth conditions and treatments}

The ecotypes Col-0 and Ler of Arabidopsis thaliana were used in the experiments as a control. The mutants tir1-1 [28], pin1 PIN1:GFP S1A, pin1 PIN1:GFP S3A, pin1 PIN1:GFP S1,3A [18], pid14/+ wag1 wag2, pid14 wag2 [19], pin3-3 [20], pin4-3 [24], pin4 pin7 and pin7-1 [29] and the line pin1 PIN1:GFP [18] were previously described. The mutants $a f b 1-3$ (SALK_070172C), afb2-3 (SALK_137151) and afb3-5 (SALK_016356C) were ordered from the SALK Institute. Seeds of the single mutants $\operatorname{lax} 2$ and $\operatorname{lax} 3$ were kindly provided by Malcolm J. Bennett (University of Nottingham, Nottingham LE12 5RD, UK) and aux1-T (SALK_020355) and lax1 (SALK_039003C) were obtained from the Nottingham Arabidopsis Stock Centre (NASC - UK). Seeds of the double and triple mutants aux1 lax1, aux1 lax2, aux1 lax3 and aux1 lax1 lax2 and the lines LAX1::LAX1:VENUS and LAX2::LAX2:VENUS were kindly provided by Ranjan Swarup (University of Nottingham, Nottingham LE12 5RD, UK). The constructs TIR1::GUS, AFBs::GUS [30], PIN1::PIN1-GFP [31], PIN3::PIN3-GFP [32], PIN4::PIN4-GFP, PIN7::PIN7-GFP [33], PID::PID-YFP [17] and WAG1::WAG1-GFP [19] have also been reported. The lines LAX1::LAX1:VENUS and LAX2::LAX2:VENUS are heterozygous for the insertion. AUX1::AUX1-YFP [34]; aux1 lax1 lax2 lax3 [35] and LAX3::LAX3-YFP [26] and all the other mutants and lines have been previously characterized [35,26]. A list of the mutants used in the present work, including the impacted gene and a description of the main phenotype observed in previous studies, is available on Suppl. Table 1.

Seed sterilization and culture were carried out according to [36,37]. Surface sterilized seeds of Col-0 were placed on medium $0.1 \mathrm{x}$ MS supplemented with $2 \%$ sucrose and solidified with agar $(0.6 \% \mathrm{w} / \mathrm{v})$ in $24-$ well microplates of $2 \mathrm{~mL}$ each. The plates were wrapped with aluminum foil and maintained in the dark for 5 days at $20 \pm 2{ }^{\circ} \mathrm{C}$ to allow for germination and etiolation. Subsequently, $0.5 \mathrm{~mL}$ of liquid $0.3 \%$ agar medium with or without auxins was aseptically added to each well, covering part of the hypocotyl. The treatment with exogenous auxin was carried out with $57 \mu \mathrm{M}$ of IAA, a concentration that promotes adventitious rooting without causing major impacts on root length (previously described by $[36,37]$ ). Exogenous supply of IAA was used aiming to synchronize and intensify the rooting process, as well as verify if it would recover the wild-type phenotype in the mutants. Stocks were freshly prepared, dissolved in $10 \mathrm{mM} \mathrm{NaOH}$, diluted in water and added to the media at the indicated concentration before autoclaving. All media $\mathrm{pH}$ were adjusted to 5.8 prior to autoclave sterilization. Seedlings were then transferred to light, with a photoperiod of $16 \mathrm{~h}$ per day and $45 \mu \mathrm{mol} \mathrm{m} \mathrm{m}^{-2} \mathrm{~s}^{-1}$ of photosynthetically active radiation (PAR) [36]. Numbers of ARs were scored at four and eight days after auxin treatment and transfer to light. The experimental system is schematically represented in Fig. 1A, indicating treatments and sampling time points.

\subsection{Confirmation of mutant identity}

To check the aux1 mutation in double and triple mutants, the seedlings were tested for auxin resistance as previously described [38] and all tested seedlings were auxin-resistant. The mutations in LAX1, LAX2 and LAX3 in the double and triple mutants and in the single mutants lax 2 and lax3 were verified by PCR according to [35]. The single mutant $\operatorname{lax} 1$ was analyzed by PCR for the absence of the wildtype fragment using the following primers: LB 5' CTTGGACCAATCAT TAATGGC $3^{\prime}$ and RB $5^{\prime}$ TCCATGGTCAGGTATGTCCTC 3 '. To verify the presence of the T-DNA in $\operatorname{lax} 1$, the RB primer listed above was used with LBb1.3 5' ATTTTGCCGATTTCGGAAC 3'. To select for the triple homozygous mutant seedlings in the progeny of pid14/ + wag1 wag2 only seedlings without cotyledons were considered in the evaluations [19]. The mutants pin3-3, pin4-3, pin4 pin7, pin7-1 and afb1-3, afb2-3, $a f b 3-5$ were analyzed by PCR for the absence of the wild-type fragment and presence of the T-DNA fragment. Primer sequences are listed on Suppl. Table 2. pin1 PIN1:GFP S1A, pin1 PIN1:GFP S3A, pin1 PIN1:GFP $S 1,3 A$ [18] and pid14/ + wag1 wag2 [19] were genotyped as previously described.

\subsection{Confocal microscopy}

For localization analysis of yellow fluorescent protein (YFP), VENUS and green fluorescent protein (GFP), the signal was visualized in water without fixation. The seedlings were immediately observed after harvesting, using a $20 \mathrm{x}$ or $40 \mathrm{x}$ long working distance water immersion objective. Signals were detected with an upright confocal laser scanning microscope Zeiss LSM 5 Exciter/AxioImager. The free software Icy [39] 


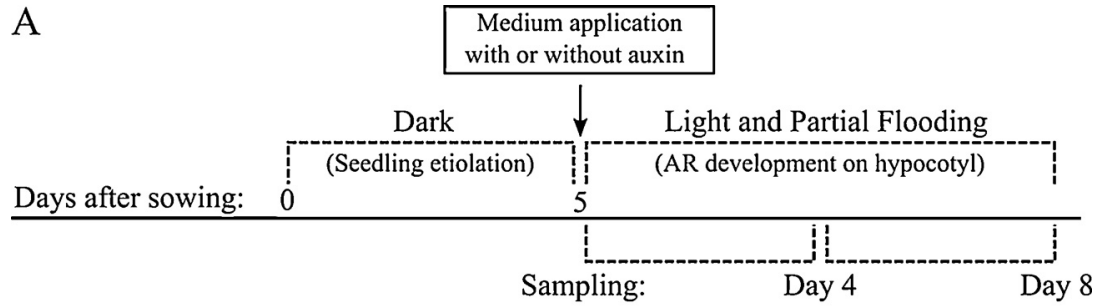

B
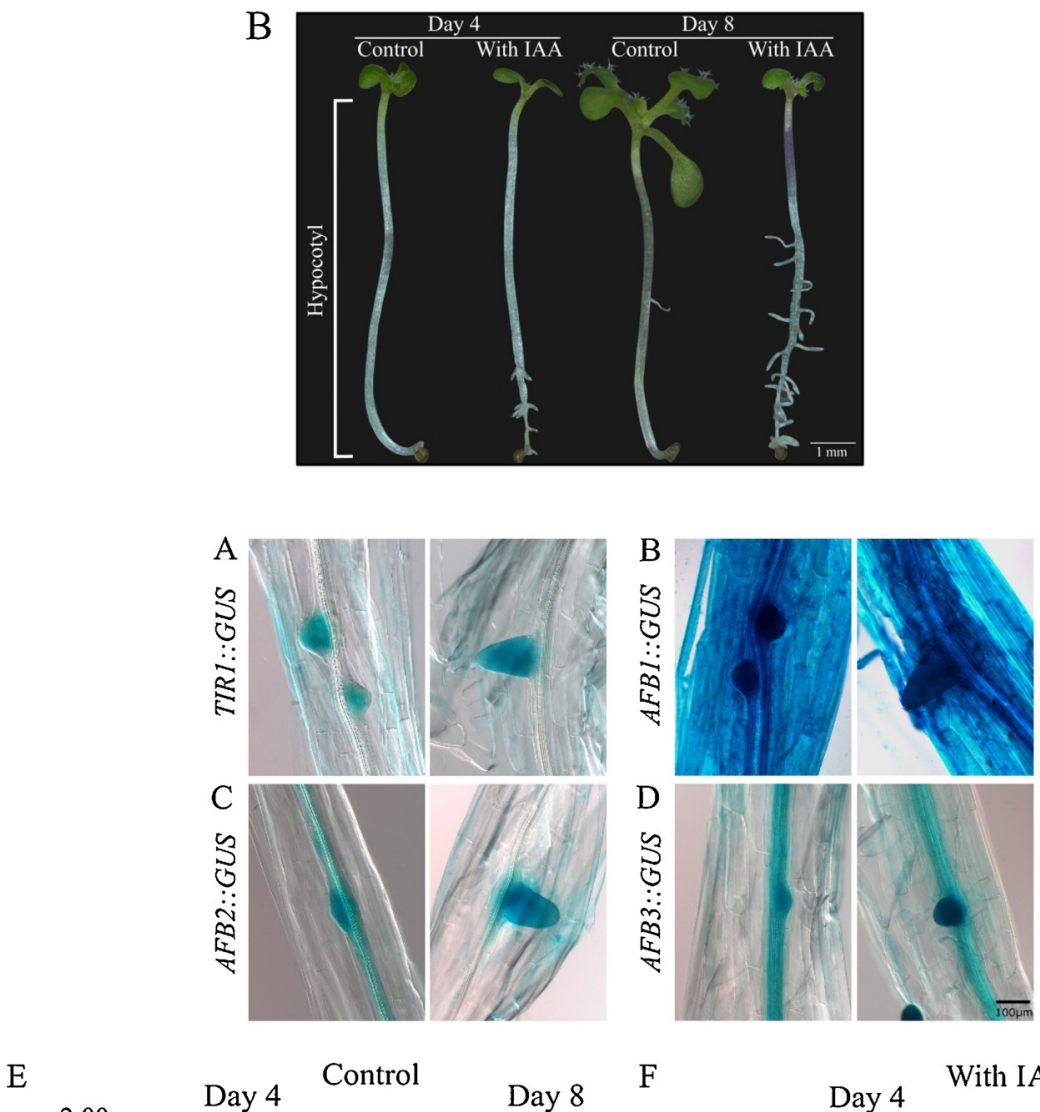

F Day 4 With IAA

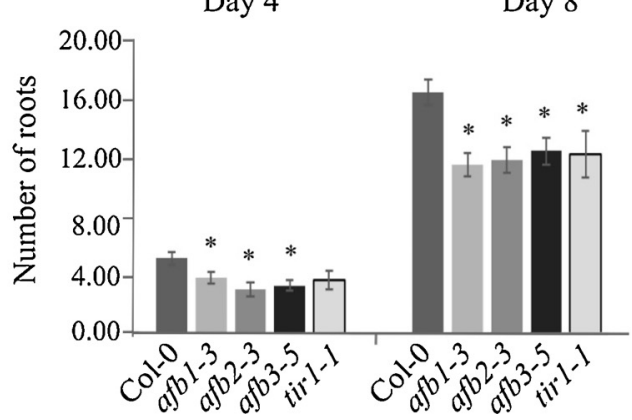

Fig. 1. Induction of adventitious roots (ARs) in hypocotyls of Arabidopsis thaliana seedlings. A) Schematic representation of the pre-etiolated and flooded system used to induce AR formation in the hypocotyl, indicating treatments and sampling time points. B) Col-0 ecotype AR development in the control (without exogenous auxin) and in the presence of exogenous auxin (57 $\mu \mathrm{M}$ IAA) at 4 and 8 days after flooding medium application on pre-etiolated seedlings. For clarity, the images show only the shoot of the seedlings (primary and lateral roots were omitted). Scale bar represents $1 \mathrm{~mm}$.

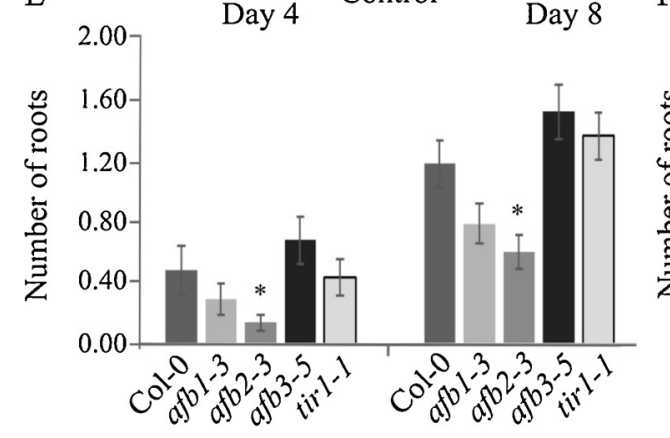

was used to select and assemble the $\mathrm{Z}$ stack slices.

\subsection{GUS ( $\beta$-glucuronidase) staining and analysis}

To detect GUS localization, the seedlings were collected, fixed in $90 \%$ acetone at $-20{ }^{\circ} \mathrm{C}$ for $20 \mathrm{~min}$, washed two times for $10 \mathrm{~min}$ each in $10 \mathrm{mM}$ EDTA, $0.1 \mathrm{M}$ sodium phosphate $(\mathrm{pH} 7.0), 2 \mathrm{mM} \mathrm{K}_{3} \mathrm{Fe}(\mathrm{CN})_{6}$ under vacuum and stained for up to $5 \mathrm{~h}$ in $10 \mathrm{mM}$ EDTA, $0.1 \mathrm{M}$ sodium phosphate $(\mathrm{pH} 7.0), 2 \mathrm{mM} \mathrm{K}_{3} \mathrm{Fe}(\mathrm{CN})_{6}, 1 \mathrm{mM} \mathrm{K}_{4} \mathrm{Fe}(\mathrm{CN})_{6} \cdot 3 \mathrm{H}_{2} \mathrm{O}$ containing $1 \mathrm{mg} \cdot \mathrm{ml}^{-1} 5$-bromo-4-chloro-3-indolyl- $\beta$-D-glucuronide at $37^{\circ} \mathrm{C}$ in the dark. The staining was interrupted by immersion in acetic acid/ ethanol (3:1) for $60 \mathrm{~min}$. Seedlings were then rehydrated in an ethanol series, and prepared in slides with chloral hydrate solution for
Fig. 2. GUS localization in ARs of 3 day-old pre-etiolated flooded seedlings containing A) TIR1::GUS, B) AFB1::GUS, C) AFB2::GUS and D) AFB3::GUS in the presence of $57 \mu \mathrm{M}$ of IAA. Scale bar represents $100 \mu \mathrm{m}$. Development of adventitious roots (ARs) in hypocotyls of auxin receptor mutants on days 4 and 8. E) Control, development of ARs without exogenous IAA. F) Development of ARs in the presence of exogenous auxin $(57 \mu \mathrm{M}$ IAA). The error bars represent the standard error. The presence of an asterisk on top of a bar indicates significant difference compared to the respective Col-0 values ( $t$-test, $p \leq 0.05$ ). 
biological replicate was composed of approximately ten seedlings.

\section{Results}

As previously reported, although it was possible to record the development of some ARs in the hypocotyl of pre-etiolated Col-0 seedlings flooded with medium devoid of auxin, application of IAA stimulated AR development (Fig. 1B) [37].

\subsection{TIR/AFB proteins play a role in AR development}

Expression of the TIR1/AFB genes during AR development was assessed using the corresponding TIR1::GUS, AFB1::GUS, AFB2::GUS and $A F B 3:: G U S$ promoter::GUS fusions. Imaging and analysis of the roots were done 3 days after transfer of seedlings to light and partial submergence in medium without or with auxin. At this timepoint the first AR primordia emerged from the etiolated hypocotyls in auxin medium. For every reporter, a strong GUS signal was observed in AR primordia (Fig. 2A-D). The signal of TIR1 and AFB2 (Fig. 2A and C, respectively) was mainly concentrated in the root primordia. Nevertheless, when primordia were smaller, some signal in the pericycle and vascular cylinder of AFB2::GUS seedlings was also visible (Fig. 2C). AFB1 was observed not only in the root primordia but in the other tissues of the hypocotyl as well (Fig. 2B). Besides being visible in the primordia, the signal of $A F B 3:: G U S$ was also found in the pericycle and vascular cylinder (Fig. 2D). In spite of the similar localization profile of AFB2::GUS and AFB3::GUS, the latter was present more evenly and with higher intensity. Based on these localization patterns we expected all four auxin co-receptors to act redundantly in AR.

To investigate their participation, we checked the single afb1-3, afb2-3, afb3-5 and tir1-1 mutants for alterations in the number of ARs on days 4 and 8 after transfer to light with or without exogenous IAA application. A mutation in AFB1 caused a reduction in AR numbers only in presence of exogenous auxin but at both timepoints evaluated (Fig. 2E and F). A similar profile was observed for the afb3-5 mutant. The $a f b 2$ mutant showed reduced adventitious roots in both time points and auxin conditions (Fig. 2E and F), whereas the tir1-1 mutant only showed a decrease in AR formation on day 8 in presence of IAA (Fig. 2E and F). These results confirm the conclusion from the localization studies that TIR1/AFB genes act redundantly in AR, but also indicate a more important role for $A F B 2$. We did not detect a clear correlation between strength of TIR/AFB promoter::GUS fusions signal and the AR defect in the corresponding mutant.

\subsection{AR development is affected in aux1-T, lax1 and lax3 mutants}

To gain insight into the role of AUX1 in adventitious root development, the localization of $A U X 1:: A U X 1-Y F P$ was examined in the presence or absence of exogenous auxin. AUX1 seemed to be expressed in the AR primordia since the first cell divisions until later stages of root development, independently of exogenous IAA supply (Fig. 3A). Besides the presence in root primordia, the AUX1-YFP signal was also observed in the pericycle and appeared to become stronger in the root tip at later stages, although it seemed to remain weaker in the other cells of the root primordia (Fig. 3A). Differences in YFP signal between the control and the treatment with exogenous IAA were not observed. Localization of $L A X 3$ was also checked, using the construct LAX3::LAX3-YFP. Unlike $A U X 1, L A X 3$ was present in stele, cortex and epidermis, but not in AR primordia (Fig. 3B). It was possible to see some signal even in the dividing pericycle cells (Fig. 3C). Exogenous IAA supply seemed to cause a stronger occurrence of LAX3 (Fig. 3B). The constructs LAX1::LAX1:VENUS and LAX2::LAX2:VENUS yielded a weak signal (Suppl. Fig. 1). Furthermore, no signal was detected in the control seedlings not treated with exogenous IAA. The localization of LAX1::LAX1:VENUS was concentrated in the stele region of the AR, whereas LAX2 presence was restricted to the central part of the AR primordia (Suppl. Fig. 1). These results indicate that AUX1 and LAX3 could play more relevant roles in adventitious rooting than LAX1 and LAX2.

In line with these observations, the aux1-T mutant showed important reductions in the number of ARs in both of the time points analyzed, even in presence of exogenous auxin (Fig. 3D and E). However, the lax 3 mutant did not show reduction in the number of ARs when compared to WT; in fact, on day eight in the absence of exogenous IAA, lax3 showed an increase in AR number compared to WT (Fig. 3D). Similarly, the lax1 mutant yielded higher number of ARs without external auxin on day 4 and with auxin on day 8, whereas lax2 did not yield any differences in AR number in the experiments (Fig. 3D and E). The exogenous supply of IAA did not cause major effects on AR development in the single mutants compared to WT, except for eliminating the higher number of roots in $\operatorname{lax} 1$ and $\operatorname{lax} 3$ on day 4 and day 8 , respectively (Fig. 3E). The double mutants aux1 lax1 and aux1 lax2 showed that mutations in LAX1 and LAX2 were not able to enhance the aux1 phenotype, unlike aux1 lax3 (Fig. 3F). The aux1 lax3 double mutant yielded the most severe reductions in AR development, completely blocking AR formation on day four and reaching the lowest root numbers on day eight without IAA (Fig. 3F). The quadruple mutant aux1 lax1 lax2 lax3 also showed severe impact on adventitious rooting in the absence of exogenous auxin (Fig. 3F). Exogenous IAA supply allowed the mutants to form as many roots as WT, except for the triple mutant aux1 lax1 lax2 on day 4 (Fig. 3G). The mutant analyses confirmed that AUX1 is a key transporter for AR initiation and development, whose role cannot be replaced by exogenous IAA supply. Also, different combinations of AUX1 mutations in conjunction with LAX1, LAX2 and LAX3 lead to important reductions in AR number, which in turn could be reversed by supplying exogenous auxin.

\subsection{PIN1, 3, 4 and 7 efflux carriers and their involvement in the adventitious rooting process}

In an attempt to determine which PIN proteins are involved in auxin distribution during the initial steps of AR development, the localization patterns of PIN1::PIN1-GFP, PIN3::PIN3-GFP, PIN4::PIN4-GFP and PIN7::PIN7-GFP were compared. PIN1 was observed at very early stages on AR primordia and remained in the primordia tissues afterwards (Fig. 4A). At later stages of primordia development, the localization was observed in the stele and root cap (Fig. 4A). PIN3::PIN3-GFP signal was stronger in the vascular cylinder and pericycle during early steps of the primordia development (Fig. 4B). As the primordia became larger, the signal became stronger in the columella precursors as well (Fig. 4B). In both PIN1::PIN1-GFP and PIN3::PIN3-GFP, differences between seedlings treated or not with exogenous IAA were not observed (Fig. 4A and B). PIN7::PIN7-GFP yielded a weak signal in the hypocotyl at an early developmental stage and in the region of provascular cells at more advanced stages of primordia development (Fig. 4C). No signal was detected in PIN4::PIN4-GFP (data not shown). The localization patterns point to a more significant participation of PIN1 and PIN3 compared to PIN4 and PIN7 in auxin transport during adventitious rooting.

To test the role of the PIN efflux carriers in AR development, we assessed the AR number in pin3-3, pin4-3, pin7 and pin4pin7 in our preetiolated and flooded hypocotyl model. The role of pin 1 could not be tested because of difficulties in selecting homozygous mutants among the etiolated seedlings. However, the role of PIN1 was tested later in relation to its phosphorylation by AGC kinases (see 3.4). The pin3-3, pin4-3 and pin4pin7 mutants are in Col-0 ecotype background and pin7 in Ler ecotype. The only Col-0 background mutant to show significantly reduced number of ARs in relation to the WT was pin3-3, when treated with exogenous auxin on day 4 (Fig. 4E). Without exogenous auxin supply, only pin4-3 of the same background ecotype developed more roots than the WT on day 8 (Fig. 4D). The double mutant pin4pin7 did not show an AR phenotype (Fig. 4). The mutation in PIN7 was more stable along the evaluations, developing more ARs than the Ler wildtype control, with statistically significant differences between day 8 
A

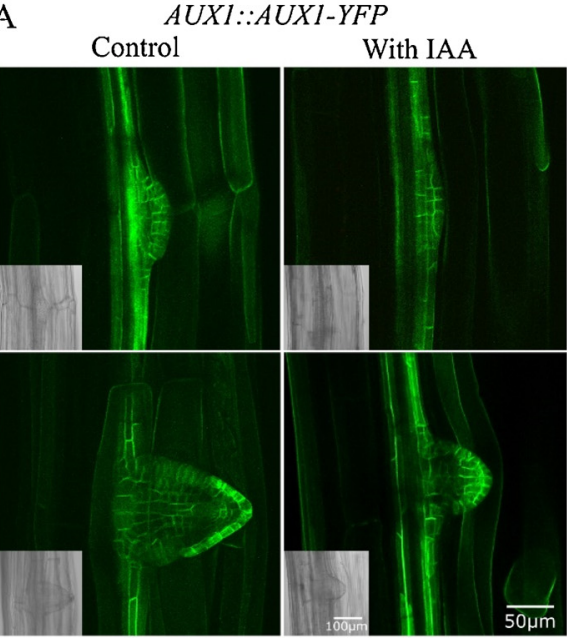

B

LAX3::LAX3-YFP

Control
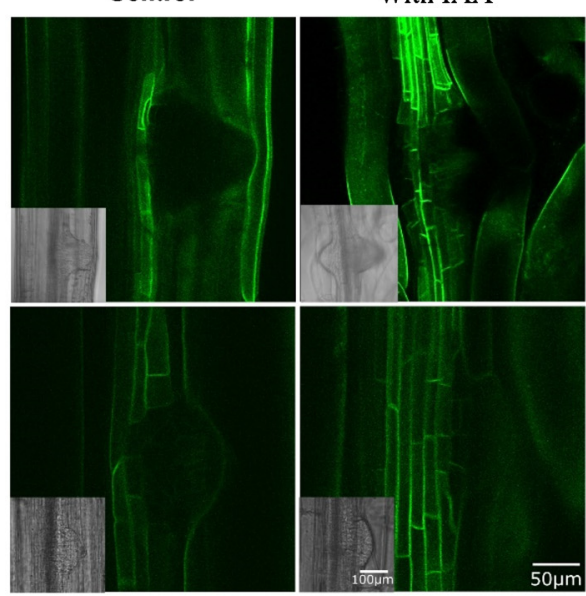

C

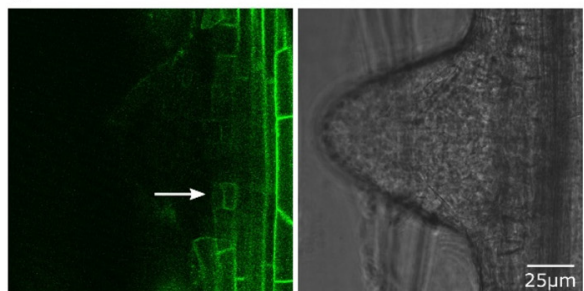

$\mathrm{D}$

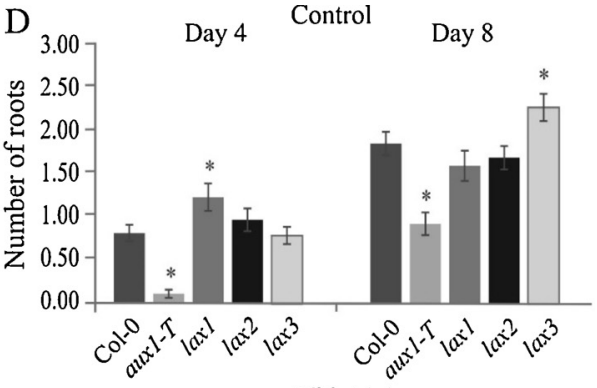

$\mathrm{E}$

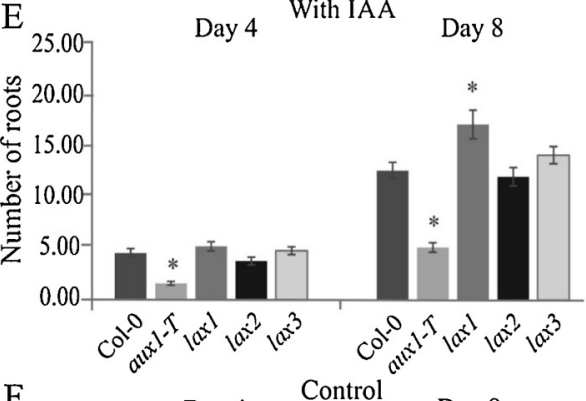

$\mathrm{F}_{3.00} \quad$ Day 4 Control Day 8

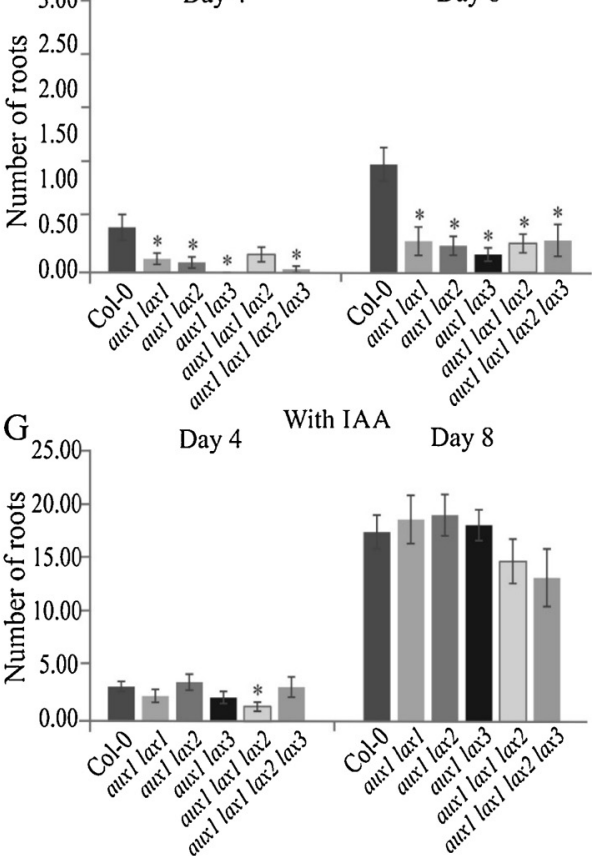

Fig. 3. Confocal imaging of pre-etiolated flooded seedlings expressing A) AUX1::AUX1YFP and B) LAX3::LAX3-YFP during AR development with or without exogenous auxin application 3 days after transfer to light. Scale bar represents $50 \mu \mathrm{m}$ on YFP images and $100 \mu \mathrm{m}$ on white field images. C) Localization of $L A X 3:: L A X 3-Y F P$ in stele and pericycle dividing cells (arrow) during AR development with exogenous IAA supply 3 days after transfer to light. Scale bar represents $25 \mu \mathrm{m}$. Adventitious roots (ARs) formation in hypocotyls of auxin influx transport single mutants on day 4 and day 8. D) Control, development of ARs without exogenous IAA. E) Development of ARs in the presence of exogenous auxin (57 $\mu \mathrm{M}$ IAA). Adventitious roots on double, triple and quadruple auxin influx transport mutants on day 4 and day 8. F) Control, development of ARs without exogenous IAA. G) Development of ARs in the presence of exogenous auxin (57 $\mu \mathrm{M}$ IAA). The error bars represent the standard error. The presence of asterisk on top of a bar indicates significant difference compared to Col-0 ( $t$-test, $p \leq 0.05$ ) control and day 4 with IAA (Fig. 4D and E). The AR analyses of PIN efflux carriers PIN3, PIN4 and PIN7 mutants did not show a constant phenotype either in the absence or the presence of exogenous auxin supply. However, the results indicated that mutations in PIN4 and PIN7 were able to somehow enhance AR numbers at specific timepoints and treatments.

\subsection{Phosphorylation of PIN efflux transporters is important for AR development}

The localization of PID and WAG1 was verified using the constructs PID::PID-YFP and WAG1::WAG1-GFP during AR development in 5-dayold pre-etiolated flooded seedlings. The signal of PID::PID-YFP was weak in the control and a bit stronger with exogenous IAA supply (Fig. 5A). WAG1::WAG1-GFP signal did not differ between the seedlings treated with IAA and those without auxin (Fig. 5B). PID and WAG1 were predominantly present in the epidermis and root cap. Before and shortly after the root protrusion, it was possible to see some localization of WAG1::WAG1-GFP in the epidermis and at the base of the primordia, when the root is being formed (Fig. 5C), though only in the seedlings with exogenous IAA treatment. These localization data suggest the participation of PID, WAG1 and WAG2 kinases in adequate PIN localization and AR formation.

To check the significance of the phosphorylation in PIN efflux transporters during adventitious rooting, we tested some previously described loss-of-phosphorylation mutants. The pid14 wag1 wag2 triple mutant lacks the three AGC kinases PID, WAG1 and WAG2 that phosphorylate three conserved serines in TPRXS motifs in PINHL and determine PIN polarity [19]. This mutant has a fully penetrant no-cotyledon phenotype in the triple homozygous seedlings [19]. Triple mutant seedlings without cotyledons were selected from a segregating population for the evaluations. In our analysis, pid14 wag1 wag2 failed to produce AR in the control on day 4 and showed a severe reduction in the number of roots on day 8 (Fig. 5D). Even when exogenous auxin was provided, a significant reduction in AR development was observed. (Fig. 5E). To test whether the marked loss of AR production was caused 


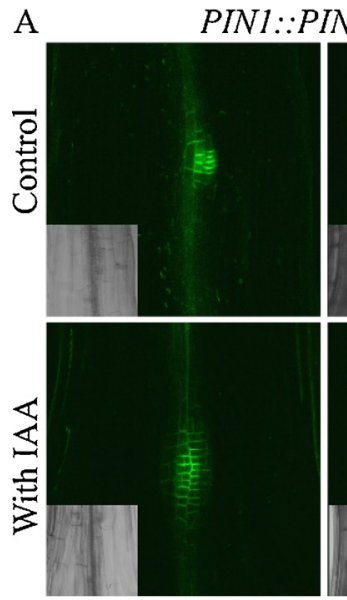

$\mathrm{D}$

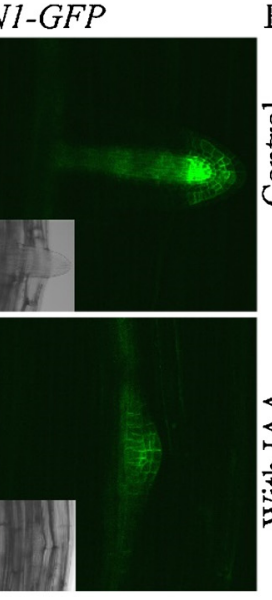

Control B PIN3::PIN3-GFP
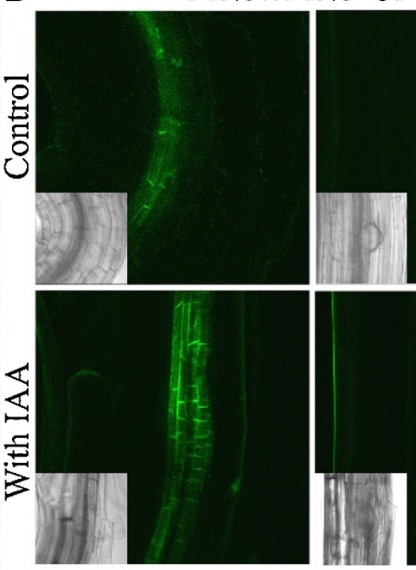

$\mathrm{E}$

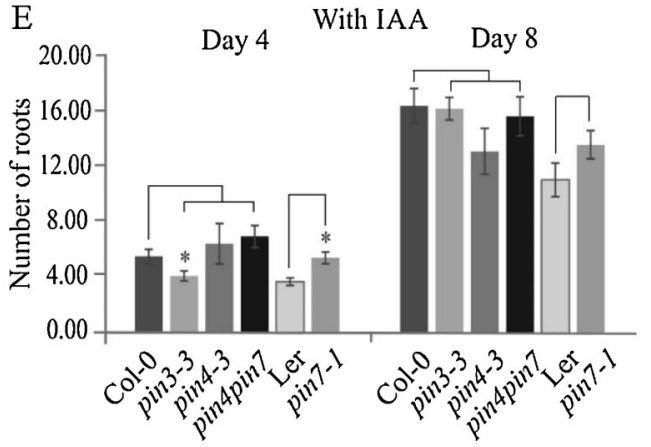

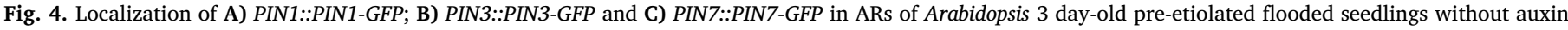

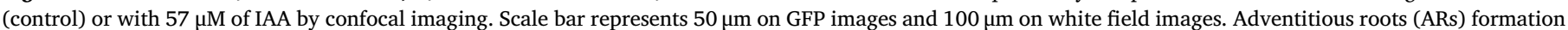

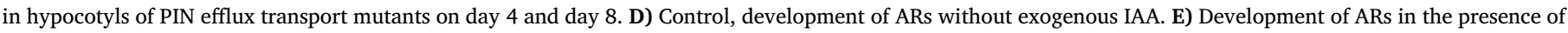

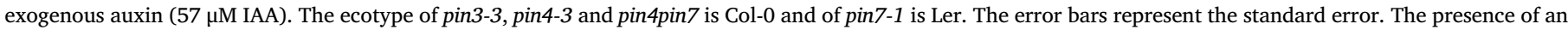
asterisk on top of a bar indicates significant difference compared to the respective ecotype ( $t$-test, $p \leq 0.05)$.

by the lack of cotyledons, seedlings without cotyledons in the progeny of the double mutant pid + wag2 were used. In this progeny, penetrance of cotyledons defects is about $50 \%$ and, of these, about $14 \%$ lack cotyledons [19]. Without exogenous auxin there was no production of ARs, differently from Col-0 (data not shown). However, with exogenously supplied IAA, the number of roots did not differ from WT (Suppl. Fig. 2), indicating that the AR capacity loss in pid14 wag1 wag2 was a result of kinase loss-of-function and not the absence of cotyledons. The loss of kinases in the triple mutant made the plant almost unable to produce ARs, highlighting the important participation of PID, WAG1 and WAG2 in adventitious rooting.

The loss-of-phosphorylation PIN1:GFP (Ser to Ala) mutants pin1 PIN1:GFP S1A, pin1 PIN1:GFP S3A and pin1 PIN1:GFP S1,3A were checked for AR development. The line pin1 PIN1:GFP was used as a control. The mutants showed reduced AR production compared to pin1 PIN1:GFP at least in one timepoint. In the case of pin1 PIN1:GFP S1A and pin1 PIN1:GFP S3A, there was an increase in the number of ARs on day 8 in the absence of exogenous auxin (Fig. 5D). When exogenous IAA was added, AR numbers were significantly lower for PIN1:GFP S3A on day 4 and PIN1:GFP S1A on day 8 compared to pin1 PIN1:GFP (Fig. 5E). The double mutant pin1 PIN1:GFP S1,3A also showed a reduction in the amount of AR with exogenous auxin supply at both sampling times (Fig. 5E). Together with the kinase mutant data, this indicates that PIN1 and also its phosphorylation are important for efficient AR development.

\section{Discussion}

The influence of auxin receptors in AR development is not yet fully understood. TIR1/AFB proteins are considered bona fide auxin receptors. The analysis of adventitious rooting in the TIR1- and AFBsdeficient seedlings indicated that all of these proteins play a role at some point during the process. However, AFB2 seemed to have a more significant role. afb2-3 mutants showed reduced AR at all timepoints and IAA conditions evaluated, and AFB2 localization was restricted to the root primordia, along with that of TIR1 (Fig. 1). Recently, Lakehal and coworkers [7] showed that TIR1 and AFB2 modulate JA homeostasis, regulating AR initiation. Some lines of evidence indicate that TIR1/AFBs have overlapping and redundant functions in plant development [30], and this seems to apply to adventitious rooting as well.

As previously observed for LRs [25], the activity of the auxin influx carrier AUX1 was crucial for adequate AR establishment (Fig. 3). In contrast, its homolog LAX3 did not seem to be essential in this process, in spite of also being inducible by exogenous IAA as shown for LRs. In fact, in one instance (day 8 without IAA), the absence of LAX3 even stimulated AR (Fig. 3D). This relative lack of importance of LAX3 in AR development differs from that observed for LRs, in which lax3 mutants showed a reduction of $40 \%$ in the number of emerged LRs in relation to the control and the result was comparable to aux1 [26]. In agreement with our data, lax 3 mutants did not show changes in adventitious rooting in Arabidopsis hypocotyls, whereas AUX1 was essential for this developmental process [40]. LAX1 and LAX2 do not seem to play essential roles on AR production. Lack of LAX1 was even beneficial to rooting without auxin on day 4 and with IAA on day 8 (Fig. 3D and E). Previous findings indicated that LAX2 regulates vascular development [14] and LAX1 and LAX2 are necessary for leaf phyllotaxy [35], but none of them has been directly related to lateral root development $[14,26]$.

Generally, the double, triple and quadruple mutations caused reductions in the number of roots without exogenous auxin, as clearly 

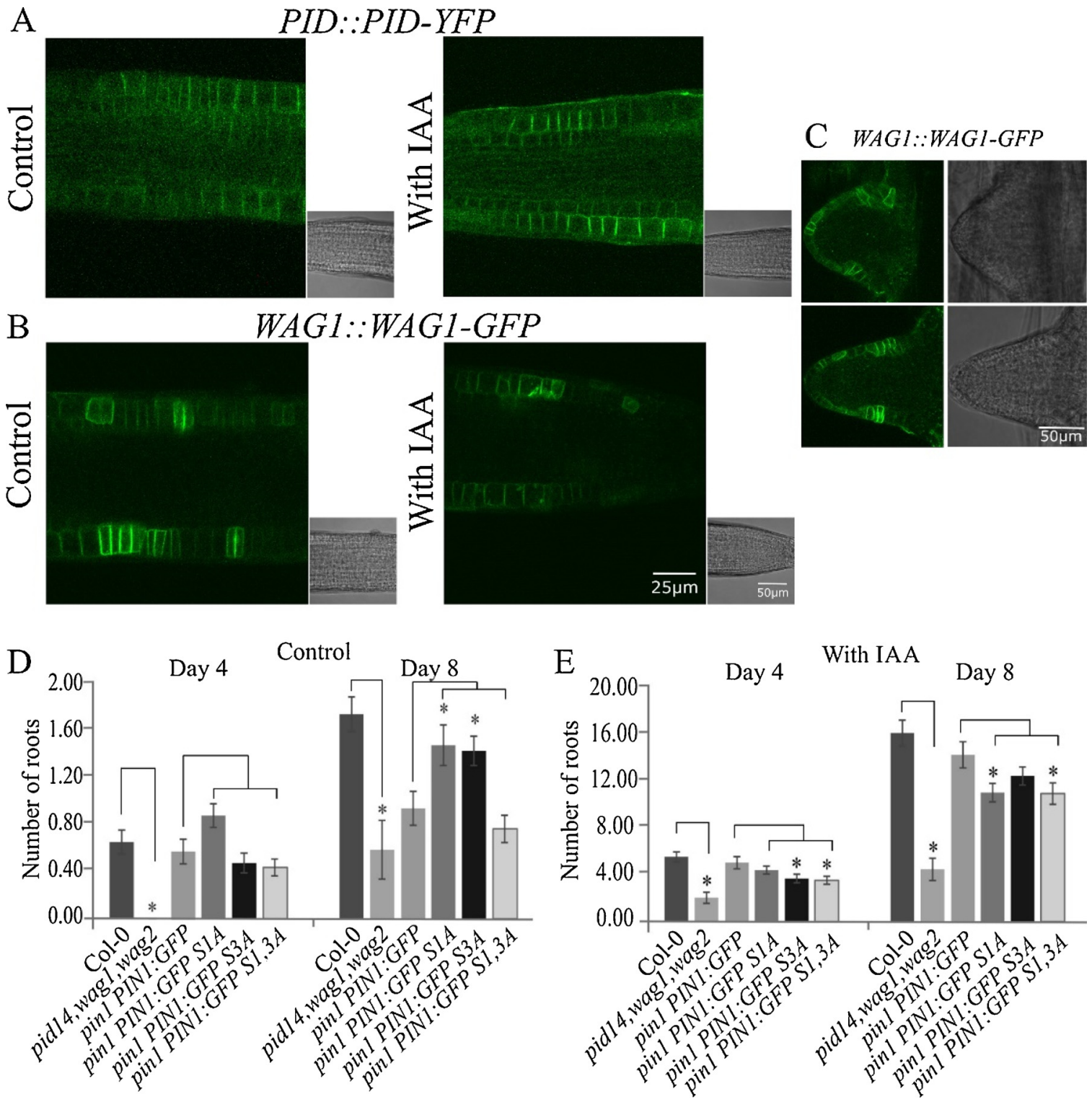

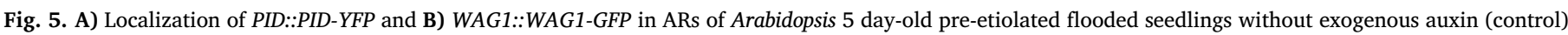

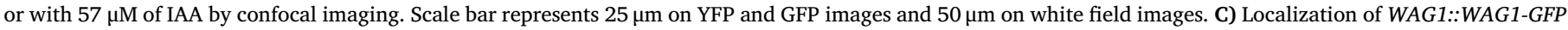

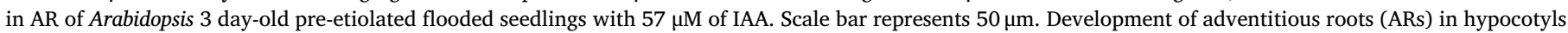

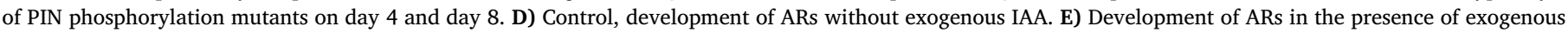

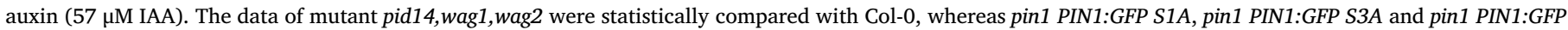

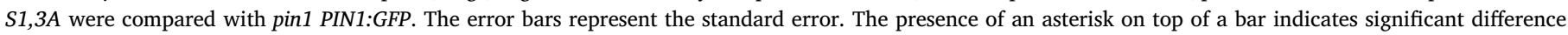
compared to the respective ecotype values ( $t$-test, $p \leq 0.05$ ).

observed on day eight. However, mutations in $L A X 1,2,3$ could compensate for aux 1 loss-of-function in the presence of exogenous IAA (Fig. 3G). This was unexpected, since exogenous supply of IAA was unable to rescue the phenotype of the single mutant aux1-T, probably because of its low sensitivity to the auxins IAA and 2,4-dichlorophenoxyacetic acid (2,4-D) [14]. In the presence of exogenous auxin, the diffusion component of transport may prevail, in which case the absence of AUX1 and one or more of the LAX functional influx transporters would demand less energy spending in influx machinery assembly, allowing diversion of this surplus to growth and differentiation of ARs. An exception to the compensation phenotype was aux1 lax1 lax2 on day four, showing that LAX3 alone is not sufficient to compensate for the missing aux1, at least in the presence of exogenous auxin and in the first days (Fig. 3G). In this sense, a conjunct action of $A U X 1$ and $L A X 3$ is apparently needed for proper auxin influx transport and AR development. When both genes are mutated, auxin may be unable to move down from the source tissues to the lower hypocotyl target tissues where ARs develop or its distribution could be disrupted. In the case of aux1-T, auxin could be moving down slower than in Col-0. To check for this possibility, future investigations could measure IAA in the roots of mutants. Moreover, mRNA expression of the mutated genes in the mutants by RT Real Time PCR or another approach to ensure that their expression is decreased or absent could also be carried out, since we were able to identify mutations only at the DNA level through PCR.

The pattern of stronger signal of LAX3::LAX3-YFP construct in the stele differs from that observed in LR development, in which expression of the same gene was restricted to a small group of cortical and epidermal cells facing the LR primordia [26]. LAX3 controls some cell-wall remodeling enzymes, which include a subtilisin-like protease (AIR3), pectate lyase (PLA2), and a xyloglucan:xyloglucosyl transferase (XTR6), leading to the softening and separation of epidermal and cortical cells overlying primordia to allow LR emergence [26]. The auxin efflux 
carrier PIN3 acts in a coordinated fashion with LAX3 to allow the softening of only a few cells that overlay the primordia [27], thereby minimizing damage to the outer cell layers. Auxin derived from the LR primordia causes the sequential expression of PIN3 and LAX3 and this network enables the expression of LAX3 in only two cortical cell layers to induce cell wall loosening, cell separation and facilitate LR emergence [27]. Therefore, data suggest that the process of etiolation and flooding that triggers AR development in the hypocotyl system employed in the present investigation [36] activates expression of LAX3 in inner tissues and at stronger levels than those reported for LRs. The auxin signaling components ARF7, ARF19 and IAA14/SLR mediate LAX3 expression induced by auxins [26]. Expression patterns of these genes and of LAX3 in mutants affected in these genes could be investigated during AR development in an attempt to address if adventitious and lateral rooting differ at levels downstream of LAX3 induction.

The auxin efflux transporter ABCB19 (ATP-Binding Cassette B19) plays a role driving AR formation in Arabidopsis hypocotyls [41]. We wondered if the participation of the PIN efflux carriers and their phosphorylation status would be important for AR development. In our observations, pid14 wag1 wag2 had a severe AR phenotype, with absence of ARs or production of only a few roots even with exogenous auxin supply (Fig. 5). The loss-of-phosphorylation PIN1:GFP (Ser to Ala) mutants pin1 PIN1:GFP S1A, pin1 PIN1:GFP S3A and pin1 PIN1:GFP $S 1,3 A$ also exhibited changes in AR development in relation to the control pin1 PIN1:GFP (Fig. 5). PID and WAG1 were observed predominantly in the root epidermis of AR primordia, similar to the localization pattern observed in LRs [19]. The results of lower AR development response for most of the loss-of-phosphorylation mutants indicate that the proper phosphorylation of PINs by the AGC kinase PID and its homologs WAG1 and WAG2 contributes to efficient AR formation. Interestingly, the higher AR response of pin1 PIN1:GFP S1A and pin1 PIN1:GFP S3A mutants under control conditions on day 8 suggests that different degrees of change in PIN phosphorylation may yield distinct AR phenotypic outcomes. In addition, these mutations may provide interesting candidate target genes for manipulation aiming to improve AR production rates in economically important species. Not only phosphorylation seems to be important for adventitious rooting, but also PIN recycling by the exocyst in Arabidopsis hypocotyls [42].

The central role of auxins and auxin transport in AR development has been a frequent topic of investigation. PIN-driven polar auxin transport seems to be required for adequate root formation. PIN1::PIN1GFP expressing seedlings showed strong signal in the root primordia, initially in the pericycle cells of the hypocotyl and later in the provascular and columella cells of the primordia (Fig. 4A). In a different adventitious rooting system, it was observed that the pin1-1 mutant had a reduction of $40 \%$ in AR formation in relation to the WT [42]. This result is in agreement with the localization pattern observed in our system, indicating that the PIN1 efflux transporter is important for primordia establishment and development. A model showing the auxin and cytokinin dynamics and participation of auxin related genes, such as AUX1, LAX1 and PIN1, in the quiescent center establishment during AR formation was proposed [43]. The visualization of PIN3 in important cells involved in primordia formation and initial development (Fig. 4B) and the reduction of ARs on day 4 in the presence of auxin (Fig. 4E) suggest that this protein is playing a role in auxin efflux transport and helping the adventitious rooting process. We could not observe clear differences between the localization of PIN3 in seedlings treated or not with exogenous auxin, unlike what has been reported in LRs cortical cells [27]. This could be due to differences in experimental conditions or represent a difference between lateral and adventitious root development.

Overall, PIN4 did not seem to have a major participation in AR development at the timepoints evaluated using the pre-etiolated flooded seedling system to induce AR formation. Localization of PIN7::PIN7-GFP was weak in the hypocotyl during primordia formation, but became more perceptible when the primordia were larger, in provascular cells. PIN3 is expressed in young emerging LRs, but has weaker expression in older roots and this happens in opposite fashion for PIN4 and PIN7, which have stronger expression in older roots [44]. Thus, the inability of LRs to grow vertically downward was attributed to an early repression of PIN4 and 7, causing a deficiency in auxin transport. It is possible that in the case of adventitious rooting, PIN4 and PIN7 display a later pattern of distribution. However, the mutants pin4-3 and pin7-1 had higher AR development in the absence of exogenous auxin, indicating that they may act as inhibitors of this developmental process, possibly by disrupting auxin concentration gradients and maxima relevant for AR. Such observations may also make these efflux transporters interesting putative targets for silencing and improvement of AR development in species of economic relevance.

Exogenous application of IAA was intended as a means of stimulating $\mathrm{AR}$ production in the various experiments described herein. However, it is well known that auxin triggers transcriptional responses that affect different aspects of plant development [12,13]. Thus, some of the observed AR responses could be partially affected by transcriptomic changes that might vary in WT or among mutants. Obviously, mutations that cause disruption in some point of the auxin activated pathway could impact the expression of genes that are also related to auxin signaling. Some mutants might show a faster or slower response to applied auxin in relation to WT, which could help explain the cases of variation in AR number at 4 or 8 days after IAA supply. However, in spite of this potential limitation that is not easily circumvented, these experiments provide important insights on the role of the various mutated proteins and the kinetics of AR response with and without an external auxin supply.

\section{Conclusions}

To sum up, although the participation of auxin receptors seems to be of fundamental importance to AR development and their action is redundant and complementary, a possible major role of AFB2 became apparent. The findings also support a fundamental role of AUX1 in adventitious rooting and provide insights on possible molecular differences between adventitious and lateral rooting. Further investigations on LAX3 participation during adventitious rooting could help to elucidate these possibilities. For adequate auxin transport and efficient formation of ARs, phosphorylation of PINs by the kinases PID, WAG1 and WAG2 seems to be essential. Finally, concerning efforts to improve AR development in economically relevant species, potential target genes for silencing (e.g. LAX1, LAX 3, PIN4, and PIN7) or overexpression (e.g. PIN1, AUX1 and AFB2) have emerged and deserve further investigation.

\section{Funding}

This work was supported by the National Council for Scientific and Technological Development (CNPq-Brazil) (grants 306079/2013-5 and 303560/2017-7) and Coordenação de Aperfeiçoamento de Pessoal de Nível Superior - (CAPES-Brazil) - Finance Code 001.

\section{CRediT authorship contribution statement}

Cibele Tesser da Costa: Data curation, Formal analysis, Investigation, Methodology, Writing - original draft. Remko Offringa: Conceptualization, Formal analysis, Funding acquisition, Project administration, Resources, Supervision, Writing - review \& editing. Arthur Germano Fett-Neto: Conceptualization, Formal analysis, Funding acquisition, Project administration, Resources, Writing - review \& editing, Supervision. 


\section{Declaration of Competing Interest}

\section{The authors declare no conflict of interest.}

\section{Appendix A. Supplementary data}

Supplementary material related to this article can be found, in the online version, at doi:https://doi.org/10.1016/j.plantsci.2019.110294.

\section{References}

[1] S. Smith, I. De Smet, Root system architecture: insights from Arabidopsis and cereal crops, Philos. Trans. R. Soc. Lond. B Biol. Sci. 367 (1595) (2012) 1441-1452.

[2] J.A. Franco, S. Bañón, M.J. Vicente, J. Miralles, J.J. Martínez-Sánchez, Root development in horticultural plants grown under abiotic stress conditions-a review, J. Hortic. Sci. Biotechnol. 86 (6) (2011) 543-556.

[3] D.I. Pacurar, I. Perrone, C. Bellini, Auxin is a central player in the hormone cross-talks that control adventitious rooting, Physiol. Plant. 151 (1) (2014) 83-96.

[4] C. Bellini, D.I. Pacurar, I. Perrone, Adventitious roots and lateral roots: similarities and differences, Annu. Rev. Plant Biol. 65 (2014) 639-666.

[5] I. Verstraeten, S. Schotte, D. Geelen, Hypocotyl adventitious root organogenesis differs from lateral root development, Front. Plant Sci. 5 (2014) 1-13.

[6] C.T. Da Costa, M.R. de Almeida, C.M. Ruedell, J. Schwambach, F.S. Maraschin, A.G. Fett-Neto, When stress and development go hand in hand: main hormonal controls of adventitious rooting in cuttings, Front. Plant Sci. 4 (2013) 133.

[7] A. Lakehall, S. Chaabouni, E. Cavel, R. Le Hir, A. Ranjan, Z. Raneshan, O. Novak, D.I. Pacurar, I. Perrone, F. Jobert, L. Gutierrez, L. Bako, C. Bellini, A molecular framework for the control of adventitious rooting by the TIR1/AFB2-Aux/IAA-dependent auxin signaling in Arabidopsis, Mol. Plant (2019), https://doi.org/10.1016/ j.molp.2019.09.001 In Press.

[8] K. Mockaitis, M. Estelle, Auxin receptors and plant development: a new signaling paradigm, Annu. Rev. Cell Dev. Biol. 24 (2008) 55-80.

[9] N. Dharmasiri, S. Dharmasiri, M. Estelle, The F-box protein TIR1 is an auxin receptor, Nature 435 (2005) 441-445.

[10] F.S. Maraschin, J. Memelink, R. Offringa, Auxin-induced, $\mathrm{SCF}^{\mathrm{TIR} 1}$-mediated polyubiquitination marks AUX/IAA proteins for degradation, Plant J. 59 (1) (2009) 100-109.

[11] A. Santner, M. Estelle, Recent advances and emerging trends in plant hormone signaling, Nature 459 (7250) (2009) 1071-1078.

[12] D. Weijers, D. Wagner, Transcriptional responses to the auxin hormone, Annu. Rev. Plant Biol. 67 (2016) 539-574.

[13] O. Leyser, Auxin signaling, Plant Phys. 176 (2018) 465-479.

[14] B. Péret, K. Swarup, A. Ferguson, M. Seth, Y. Yang, S. Dhondt, N. James, I. Casimiro, P. Perry, A. Syed, H. Yang, J. Reemmer, E. Venison, C. Howells, M.A. Perez-Amador, J. Yun, J. Alonso, G.T. Beemster, L. Laplaze, A. Murphy, M.J. Bennett, E. Nielsen, R. Swarup, $A U X / L A X$ genes encode a family of auxin influx transporters that perform distinct functions during Arabidopsis development, Plant Cell 24 (7) (2012) $2874-2885$

[15] M.E. Habets, R. Offringa, PIN-driven polar auxin transport in plant developmental plasticity: a key target for environmental and endogenous signals, New Phytol. 203 (2014) 362-377.

[16] J. Mravec, P. Skůpa, A. Bailly, K. Hoyerová, P. Křeček, A. Bielach, J. Petrášek, J. Zhang, V. Gaykova, Y.-D. Stierhof, P.I. Dobrev, K. Schwarzerová, J. Rolčík, D. Seifertová, C. Luschnig, E. Benková, E. Zažímalová, M. Geisler, J. Friml, Subcellular homeostasis of phytohormone auxin is mediated by the ER-localized PIN5 transporter, Nature 459 (2009) 1136-1140.

[17] M. Michniewicz, M.K. Zago, L. Abas, D. Weijers, A. Schweighofer, I. Meskiene, M.G. Heisler, C. Ohno, J. Zhang, F. Huang, R. Schwab, D. Weigel, E.M. Meyerowitz, C. Luschnig, R. Offringa, J. Friml, Antagonistic regulation of PIN phosphorylation by PP2A and PINOID directs auxin flux, Cell 130 (2007) 1044-1056.

[18] F. Huang, M.K. Zago, L. Abas, A. van Marion, C.S. Galvan-Ampudia, R. Offringa, Phosphorylation of conserved PIN motifs directs Arabidopsis PIN1 polarity and auxin transport, Plant Cell 22 (2010) 1129-1142.

[19] P. Dhonukshe, F. Huang, C.S. Galvan-Ampudia, A.P. Mahonen, J. Kleine-Vehn, J. Xu, A. Quint, K. Prasad, J. Friml, B. Scheres, R. Offringa, Plasma membranebound AGC3 kinases phosphorylate PIN auxin carriers at TPRXS(N/S) motifs to direct apical PIN recycling, Development 137 (2010) 3245-3255.

[20] J. Friml, J. Wiśniewska, E. Benková, K. Mendgen, K. Palme, Lateral relocation of auxin efflux regulator PIN3 mediates tropism in Arabidopsis, Nature 415 (6873) (2002) 806-809.

[21] J. Kleine-Vehn, Z. Ding, A.R. Jones, M. Tasaka, M.T. Morita, J. Friml, Gravity-induced PIN transcytosis for polarization of auxin fluxes in gravity-sensing root cells, PNAS 107 (51) (2010) 22344-22349.

[22] D.R. Lewis, S. Negi, P. Sukumar, G.K. Muday, Ethylene inhibits lateral root development, increases IAA transport and expression of PIN3 and PIN7 auxin efflux carriers, Development 138 (16) (2011) 3485-3495.

[23] F. Della Rovere, L. Fattorini, S. D’Angeli, A. Veloccia, G. Falasca, M.M. Altamura, Auxin and cytokinin control formation of the quiescent centre in the adventitious root apex of Arabidopsis, Ann. Bot. 112 (2013) 1395-1407.

[24] J. Friml, E. Benková, I. Blilou, J. Wisniewska, T. Hamann, K. Ljung, S. Woody, G. Sandberg, B. Scheres, G. Jurgens, K. Palme, AtPIN4 mediates sink-driven auxin gradients and root patterning in Arabidopsis, Cell 108 (5) (2002) 661-673.

[25] A. Marchant, R. Bhalerao, I. Casimiro, J. Eklöf, P.J. Casero, M. Bennett, G. Sandberg, AUX1 promotes lateral root formation by facilitating indole-3-acetic acid distribution between sink and source tissues in the Arabidopsis seedling, Plant Cell 14 (3) (2002) 589-597.

[26] K. Swarup, E. Benková, R. Swarup, I. Casimiro, B. Péret, Y. Yang, G. Parry, E. Nielsen, I. De Smet, S. Vanneste, M.P. Levesque, D. Carrier, N. James, V. Calvo, K. Ljung, E. Kramer, R. Roberts, N. Graham, S. Marillonnet, K. Patel, J.D.G. Jones, C.G. Taylor, D.P. Schachtman, S. May, G. Sandberg, P. Benfey, J. Friml, I. Kerr, T. Beeckman, L. Laplaze, M.J. Bennett, The auxin influx carrier LAX3 promotes lateral root emergence, Nat. Cell Biol. 10 (8) (2008) 946-954.

[27] B. Péret, A.M. Middleton, A.P. French, A. Larrieu, A. Bishopp, M. Njo, D.M. Wells, S. Porco, N. Mellor, L.R. Band, I. Casimiro, J. Kleine-Vehn, S. Vanneste, I. Sairanen, R. Mallet, G. Sandberg, K. Ljung, T. Beeckman, E. Benkova, J. Friml, E. Kramer, J.R. King, I. De Smet, T. Pridmore, M. Owen, M.J. Bennett, Sequential induction of auxin efflux and influx carriers regulates lateral root emergence, Mol. Syst. Biol. 9 (1) (2013) 699.

[28] M. Ruegger, E. Dewey, W.M. Gray, L. Hobbie, J. Turner, M. Estelle, The TIR1 protein of Arabidopsis functions in auxin response and is related to human SKP2 and yeast Grr1p, Genes Dev. 12 (2) (1998) 198-207.

[29] J. Friml, A. Vieten, M. Sauer, D. Weijers, H. Schwarz, T. Hamann, R. Offringa, G. Jürgens, Efflux-dependent auxin gradients establish the apical-basal axis of Arabidopsis, Nature 426 (6963) (2003) 147-153.

[30] N. Dharmasiri, S. Dharmasiri, D. Weijers, E. Lechner, M. Yamada, L. Hobbie, J.S. Ehrismann, G. Jurgens, M. Estelle, Plant development is regulated by a family of auxin receptor F box proteins, Dev. Cell 9 (2005) 109-119.

[31] E. Benková, M. Michniewicz, M. Sauer, T. Teichmann, D. Seifertová, G. Jürgens, J. Friml, Local, efflux-dependent auxin gradients as a common module for plant organ formation, Cell 115 (5) (2003) 591-602.

[32] P. Žádníková, J. Petrášek, P. Marhavý, V. Raz, F. Vandenbussche, Z. Ding, K. Schwarzerová, M.T. Morita, M. Tasaka, J. Hejátko, D. Van Der Straeten, J. Friml, E. Benková, Role of PIN-mediated auxin efflux in apical hook development of Arabidopsis thaliana, Development 137 (4) (2010) 607-617.

[33] I. Blilou, J. Xu, M. Wildwater, V. Willemsen, I. Paponov, J. Friml, R. Heidstra, M. Aida, K. Palme, B. Scheres, The PIN auxin efflux facilitator network controls growth and patterning in Arabidopsis roots, Nature 433 (7021) (2005) 39-44.

[34] R. Swarup, J. Kargul, A. Marchant, D. Zadik, A. Rahman, R. Mills, A. Yemm, S. May, L. Williams, P. Millner, S. Tsurumi, I. Moore, R. Napier, I.D. Kerr, M.J. Bennett, Structure-function analysis of the presumptive Arabidopsis auxin permease AUX1, Plant Cell 16 (11) (2004) 3069-3083.

[35] K. Bainbridge, S. Guyomarc'h, E. Bayer, R. Swarup, M. Bennett, T. Mandel, C. Kuhlemeier, Auxin influx carriers stabilize phyllotactic patterning, Genes Dev. 22 (6) (2008) 810-823.

[36] L.R. Correa, J. Troleis, A.A. Mastroberti, J.E. Mariath, A.G. Fett-Neto, Distinct modes of adventitious rooting in Arabidopsis thaliana, Plant Biol. 14 (2012) $100-109$.

[37] C.T. Da Costa, M.L. Gaeta, J.E.A. Mariath, R. Offringa, A.G. Fett-Neto, Comparative adventitious root development in pre-etiolated and flooded Arabidopsis hypocotyls exposed to different auxins, Plant Physiol. Biochem. 127 (2018) 161-168.

[38] A. Marchant, M.J. Bennett, The Arabidopsis AUX1 gene: a model system to study mRNA processing in plants, Plant Mol. Biol. 36 (3) (1998) 463-471.

[39] F. de Chaumont, S. Dallongeville, N. Chenouard, N. Hervé, S. Pop, T. Provoost, V. Meas-Yedid, P. Pankajakshan, T. Lecomte, Y.L. Montagner, T. Lagache, A. Dufour, J.C. Olivo-Marin, Icy: an open bioimage informatics platform for extended reproducible research, Nat. Methods 9 (7) (2012) 690-696.

[40] F. Della Rovere, L. Fattorini, S. D’Angeli, A. Veloccia, S. Del Duca, G. Cai, G. Falasca, M.M. Altamura, Arabidopsis SHR and SCR transcription factors and AUX1 auxin influx carrier control the switch between adventitious rooting and xylogenesis in planta and in vitro cultured thin cell layers, Ann. Bot. 115 (2015) 617-628.

[41] P. Sukumar, G.S. Maloney, G.K. Muday, Localized induction of the ATP-Binding Cassette B19 auxin transporter enhances adventitious root formation in Arabidopsis, Plant Physiol. 162 (3) (2013) 1392-1405.

[42] E.J. Drdová, M. Klejchová, K. Janko, M. Hála, H. Soukupová, F. Cvrčková, V. Žárský, Developmental plasticity of Arabidopsis hypocotyl is dependent on exocyst complex function, J. Exp. Bot. 70 (4) (2019) 1255-1265.

[43] F. Della Rovere, L. Fattorini, M. Ronzan, G. Falasca, M.M. Altamura, The quiescen center and the stem cell niche in the adventitious roots of Arabidopsis thaliana, Plant Signal. Behav. 11 (5) (2016) e1176660.

[44] M.R. Rosquete, D. von Wangenheim, P. Marhavý, E. Barbez, E.H. Stelzer, E. Benková, A. Maizel, J. Kleine-Vehn, An auxin transport mechanism restricts positive orthogravitropism in lateral roots, Cur. Biol. 23 (9) (2013) 817-822. 